\title{
A Novel Phellinidium sp. Causes Laminated Root Rot on Qilian Juniper (Sabina przewalskii) in Northwest China
}

Bao-Kai Cui, Yu-Cheng Dai, and Shuang-Hui He, Institute of Microbiology, Beijing Forestry University, P.O. Box 61, Beijing 100083, China; and Li-Wei Zhou and Hai-Sheng Yuan, State Key Laboratory of Forest and Soil Ecology, Institute of Applied Ecology, Chinese Academy of Sciences, Shenyang 110164, China

\section{Abstract}

Cui, B. K., Dai, Y. C., He, S. H., Zhou, L. W., and Yuan, H. S. 2015. A novel Phellinidium sp. causes laminated root rot on Qilian juniper (Sabina przewalskii) in northwest China. Plant Dis. 99:39-43.

A laminated root rot on Qilian juniper (Sabina przewalskii) caused by a species of Phellinidium (Basidiomycota) was observed in northwest China. Seventeen fungal samples collected from Qinghai Province during 2012 and 2013 were used for taxonomic and pathogenicity tests. The fungal pathogen was identified by morphological characters and phylogenetic analyses based on nLSU sequences. A new fungus is described herein as Phellinidium qilianense sp. nov. One-year-old Qilian juniper seedlings were wound-inoculated under controlled conditions to test pathogenicity of the fungal species. The fungus was successfully reisolated from decayed tissue of tested seedlings. P. qilianense is a new forest pathogen on coniferous trees in China.
Phellinidium (Kotl.) Fiasson \& Niemelä is a small genus with several species that are tree-pathogenic fungi. Two members of the Phellinidium weirii (Murrill) Y.C. Dai complex grow on living conifers: one is $P$. weirii sensu stricto (the cedar type), which is usually found on species of the Cupressaceae in North America; the other, $P$. sulphurascens (Pilát) Y.C. Dai (the Douglas-fir type) occurs on species of Pinaceae $(6,9)$. Outside North America, $P$. weirii has been reported on Juniperus spp. in the Ural Mountains (2) and P. sulphurascens has been reported on Picea, Abies, Larix, and Pinus spp. in East Asia, including China (3). Besides the above-mentioned species, Phellinidium noxium (Corner) Bondartseva \& S. Herrera is a serious pathogen on many angiosperm trees in tropical Asia $(5,10)$. In North America, P. weirii causes significant root and butt rot of western red cedar (Thuja plicata), while $P$. sulphurascens is especially damaging to Douglas-fir (Pseudotsuga menziesii) $(1,9,13,15,19)$. The pathogenicity of Phellinidium in China is poorly known.

The Qilian juniper, Sabina przewalskii Kom. (syn. Juniperus przewalskii), is an endemic species in China, and its natural distribution is around $32^{\circ} 35^{\prime}$ to $39^{\circ} 05^{\prime} \mathrm{N}$ and $96^{\circ} 26^{\prime}$ to $104^{\circ} 20^{\prime} \mathrm{E}$. The species occurs mainly as pure stands in the Qilian Mountains of Qinghai Province in northwest China, at elevations ranging from 2,600 to $2,800 \mathrm{~m}$. It is a very important tree species in western China because it is both highly drought tolerant and cold hardy and it plays an important role in preventing local soil erosion. In 2004, a fungus causing laminated root rot on S. przewalskii was found in the Qilian Mountains (2). Based on preliminary investigations, the pathogen was identified as $P$. weirii $(2,4,5)$. Because the disease has increased in severity since 2004, further research on the pathogen and disease was carried out during 2012 to 2013 and these new results are reported here.

The objectives of our study were to 1) identify the pathogenic causal fungus based on molecular and morphological analyses, 2) determine the phylogenetic relationship of the fungus with other similar species, 3) provide a detailed description of the decay on

Corresponding author: Y. C. Dai, E-mail: yuchengd@yahoo.com

Accepted for publication 7 July 2014.

http://dx.doi.org/10.1094/PDIS-03-14-0335-RE

(c) 2015 The American Phytopathological Society
Qilian juniper caused by the fungus, and 4) evaluate the pathogenicity of the fungus on Qilian juniper.

\section{Materials and Methods}

Disease surveys. Healthy stands and diseased stands of $S$. przewalskii were investigated. Symptoms of the disease included slow growth, chlorotic foliage, wilt, and mortality. After cutting some roots of the diseased trees, cambial necrosis and decay were found. Five plots in the diseased stands of $S$. przewalskii on different slopes were investigated. Each plot was $100 \times 100 \mathrm{~m}$ and all nonsymptomatic, symptomatic, and dead trees were calculated. Trees were considered infected if they had chlorotic foliage or the fruiting body of Phellinidium qilianense was found on its roots. The characteristics for laminated rot include a delamination where the wood separates at the annual rings. The decayed wood is usually soft, paler and lighter than sound wood.

Fungal collections and isolations. Seventeen fungal and/or decayed wood samples (IFP 018444, 018454, 018468, and 018481; and Dai YC 13303, 13304, 13305, 13306, 13307, 13308, 13310, $13311,13312,13315,13316,13317$, and13320) were collected from the roots of living, dying, and dead S. przewalskii trees occurring throughout the Xianmi Forest Farm, Qinghai Province, northwest China during 2012 and 2013 (Table 1). Five fungal strains (Dai YC 13304, 13308, 13311, 13315, and 13317) isolated from living, dying, or dead trees and/or fruiting bodies of the fungus (Table 1) were selected for deposition in the herbaria of the Institute of Applied Ecology, Chinese Academy of Sciences (IFP), and Beijing Forestry University (BJFC). These same fungal strains were preserved on PDA in the herbarium of the BJFC.

Morphological studies. The studied specimens were deposited at the herbaria of the BJFC and the IFP. Sections were studied at magnification up to $1,000 \times$ using a Nikon Eclipse 80i microscope and phase contrast illumination (Nikon, Tokyo). Drawings were made with the aid of a drawing tube. Microscopic features, measurements, and drawings were made from slide preparations stained with Cotton Blue and Melzer's reagent. Spores were measured from sections cut from the tubes. In presenting the variation in the size of the spores, $5 \%$ of measurements were excluded from each end of the range, and were given in parentheses. In the text, the following abbreviations were used: IKI $=$ Melzer's reagent, IKI $-=$ both inamyloid and indextrinoid, $\mathrm{KOH}$ $=5 \%$ potassium hydroxide, $\mathrm{CB}=$ Cotton $\mathrm{Blue}, \mathrm{CB}-=$ acyanophilous, $\mathrm{L}=$ mean spore length (arithmetic average of all spores), $\mathrm{W}=$ mean spore width (arithmetic average of all spores), 
$\mathrm{Q}=$ variation in the $\mathrm{L} / \mathrm{W}$ ratios between the specimens studied, and $\mathrm{n}(\mathrm{a} / \mathrm{b})=$ number of spores (a) measured from given number $(\mathrm{b})$ of specimens.

DNA extraction, PCR amplification, and phylogenetic analyses. A Phire Plant Direct PCR Kit (Finnzymes Oy, Finland) was used to perform PCR amplifications from fruiting bodies and mycelia on plates according to the manufacturer's instructions. These tissues originated from wood samples or fruiting bodies of nine isolates randomly selected from the 17 isolates we collected. About $50 \mathrm{mg}$ samples were lysed in $30 \mu \mathrm{l}$ dilution buffer for DNA extraction. After incubating $3 \mathrm{~min}$ at room temperature, $0.75 \mu \mathrm{l}$ of the supernatant were used as template for a $30 \mu \mathrm{l}$ PCR reaction. The nuclear large subunit (nLSU) region of the extracted DNA was amplified using the primers LR0R and LR7 (21). The PCR procedure was as follows: initial denaturation at $98^{\circ} \mathrm{C}$ for $5 \mathrm{~min}$, followed by 39 cycles at $98^{\circ} \mathrm{C}$ for $5 \mathrm{~s}, 48^{\circ} \mathrm{C}$ for $5 \mathrm{~s}$, and $72^{\circ} \mathrm{C}$ for 5 $\mathrm{s}$, and a final extension of $72^{\circ} \mathrm{C}$ for $10 \mathrm{~min}$. The PCR products were sequenced at the Beijing Genomics Institute, China, using the same primers as those used in our PCR procedure. All newly generated sequences are deposited in GenBank (http://www.ncbi.nlm.nih. gov; Table 1). The nLSU sequences of $P$. sulphurascens and $P$. weirii generated by Wagner and Fischer (22) and Lim et al. (14) were retrieved from GenBank to explore the phylogenetic relationships among these two species and the newly sequenced samples. Phellinidium ferrugineofuscum (P. Karst.) Fiasson \& Niemelä, the generic type, was selected as the outgroup (22) to use in our phylogenetic analysis. The nLSU dataset was aligned using MAFFT $7.110(11,12)$ with Q-INS-i option. Sequence alignment was deposited at TreeBASE (http://purl.org/phylo/treebase; submission ID 15617). The best-fit evolutionary model for the resulting alignment was estimated as GTR model using jModelTest $(8,17)$. Following this model, the maximum likelihood (ML) analysis was performed using raxmlGUI $1.2(18,20)$ with auto FC option (16) in bootstrap (BS) replicates.

Pathogenicity tests. One-year-old seedlings of S. przewalskii obtained from a nursery in the Xianmi Forest Farm, Qinghai Province, northwest China, were planted into clay pots containing a sterile standard soil mixture of peat, perlite, and vermiculite (6:2:2 ratio by volume) in a greenhouse.

Four isolates of $P$. qilianense (Dai YC 13304, 13308, 13315, and 13317) that we isolated from roots of living, dying, and dead trees of $S$. przewalskii and/or fruiting bodies of the fungus were used to wound-inoculate $36 \mathrm{~S}$. przewalskii seedlings (three seedlings in each pot, one isolate repeat in three pots). All four isolates were grown in petri dishes containing PDA for 10 days prior to inoculation. The seedlings were transplanted to pots after 1 week for inoculations, a random root was selected, and its connection part to the main root was excised using a sterile scalpel. An agar plug with fresh mycelia was cut from each culture and placed onto the wounded tissue, and the wound was sealed with Parafilm. A total of nine control seedlings were wounded using a sterile scalpel prior to inoculation with sterile PDA plugs. Inoculations were conducted in August 2013. Seedlings were monitored weekly for symptom development such as chlorotic foliage, wilt, and mortality. Root decay was also examined when chlorotic foliage appeared after 2 months. A segment of about $2 \mathrm{~mm}$ decayed root was placed in petri dishes containing PDA and incubated. Reisolations were made in petri dishes containing PDA and after growth, their colony morphology (brownish generative hyphae with simple septa and hyphoid setae with sharp tips) was observed. nLSU sequences were then analyzed and compared with those of the inoculated cultures.

\section{Results}

Disease surveys, fungal collection, and morphological characterization. Dead and dying trees of $S$. przewalskii were found in many natural forests of the Qilian Mountains, Menyuan County, Qinghai Province (Fig. 1A). The trees were about 80 to 220 years old according to the stand data from the local forest department and occurred in almost pure stands. A disease patch or root rot center up to 5 ha was found consisting of standing dead and dying symptomatic trees (Fig. 1B); some fallen trees were also
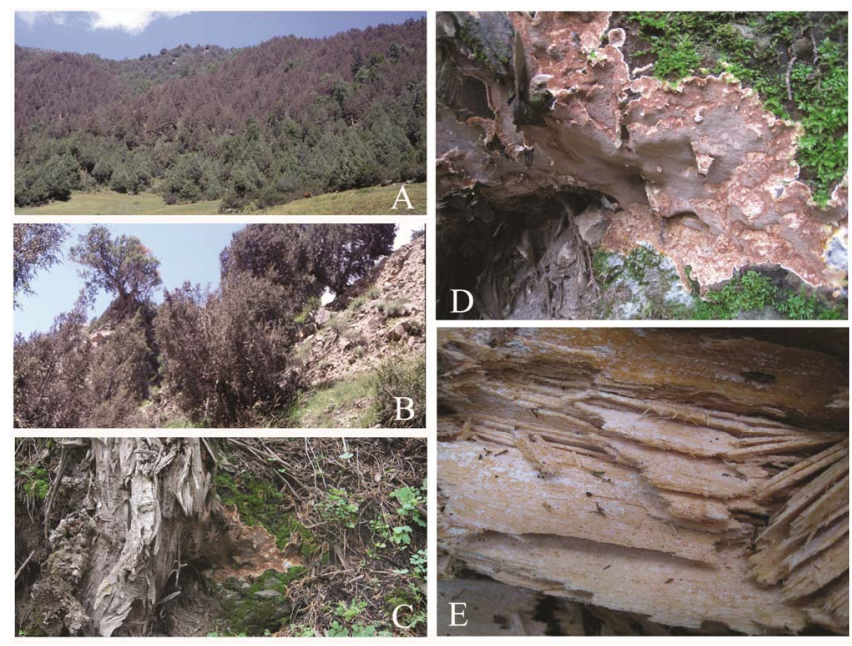

Fig. 1. A, Dead trees in pure forest of Sabina przewalskii. B, Dead and dying trees of S. przewalskii. C, The fruiting body of Phellinidium qilianense (sample Dai 13309) on root of dying S. przewalskii. D, The fruiting body of $P$. qilianense (Sample Dai 13309). E, The laminated decay on wood of S. przewalskii caused by $P$. qilianense.

Table 1. Samples of Phellinidium qilianense on Sabina przewalskii in Qinghai Province used in this study

\begin{tabular}{|c|c|c|c|c|c|}
\hline Samples & Host & Location & Year & Collector & GenBank no. (nLSU) \\
\hline IFP $18444^{\mathrm{a}}$ & Living tree & Sigou, Menyuan & 2012 & Yuan HS & KJ635808 \\
\hline IFP $18454^{\mathrm{a}}$ & Dying tree & Sigou, Menyuan & 2012 & Yuan HS & KJ635809 \\
\hline IFP $18468^{a}$ & Fallen trunk & Donghai, Menyuan & 2012 & Yuan HS & KJ635810 \\
\hline IFP $18481^{\mathrm{a}}$ & Stump & Donghai, Menyuan & 2012 & Yuan HS & - \\
\hline Dai $13303^{a}$ & Living tree & Xianfeng, Menyuan & 2013 & Dai YC & - \\
\hline Dai $13304^{b}$ & Living tree & Xianfeng, Menyuan & 2013 & Dai YC & - \\
\hline Dai $13305^{a}$ & Living tree & Xianfeng, Menyuan & 2013 & Dai YC & - \\
\hline Dai $13306^{a}$ & Living tree & Xianfeng, Menyuan & 2013 & Dai YC & - \\
\hline Dai $13307^{a}$ & Dying tree & Xianfeng, Menyuan & 2013 & Dai YC & - \\
\hline Dai $13308^{b}$ & Dying tree & Xianfeng, Menyuan & 2013 & Dai YC & - \\
\hline Dai $13310^{\mathrm{a}}$ & Dead tree & Xianfeng, Menyuan & 2013 & Dai YC & KJ635811 \\
\hline Dai $13311^{\mathrm{a}}$ & Dying tree & Xianfeng, Menyuan & 2013 & Dai YC & KJ635812 \\
\hline Dai $13312^{\mathrm{a}}$ & Dead tree & Xianfeng, Menyuan & 2013 & Dai YC & KJ635813 \\
\hline Dai $13315^{\mathrm{b}}$ & Dead tree & Xianfeng, Menyuan & 2013 & Dai YC & KJ635814 \\
\hline Dai $13316^{\mathrm{a}}$ & Dead tree & Xianfeng, Menyuan & 2013 & Dai YC & - \\
\hline Dai $13317^{b}$ & Fallen trunk & Xianfeng, Menyuan & 2013 & Dai YC & KJ635815 \\
\hline Dai $13320^{b}$ & Fallen trunk & Xianfeng, Menyuan & 2013 & Dai YC & KJ635816 \\
\hline
\end{tabular}

\footnotetext{
a Indicates fruiting body.

b Indicates isolate from wood or fruiting body.
} 
occasionally present in the patch. Symptomatic trees showed reduced branch and leader growth, thin crowns, and chlorotic foliage. Based on our investigation on the five plots in the different diseased stands located on different slopes, the infected rate ranged from 40 to $90 \%$ while mortality ranged from 20 to $70 \%$. But in another plot not included in the five plots, almost $100 \%$ of the trees were infected and mortality was approximately $80 \%$. Fungal fruiting bodies were found at the base of many dead and dying trees (Fig. 1C), mostly on the underside of roots and stems of fallen infected trees. Fruiting bodies were perennial, resupinate, and dark brown, with poroid hymenophores (Fig. 1D). In the early stage of decay, $P$. qilianense-infected wood of dead trees and fallen trunks was woody hard, then became characteristically laminated in advanced stages of decay (Fig. 1E). Microscopically, the fungus had a monomitic hyphal system, hyphae without clamp connections, tramal tissue with hyphoid setae, and thin-walled, hyaline, smooth, ellipsoid basidiospores. The fungus belongs to Phellinidium (Hymenochaetaceae, Basidiomycota), and is closely related to $P$. weirii $(3,7)$.

Phylogenetic analyses. A total of nine collections of the pathogens on S. przewalskii were nLSU-sequenced (Table 1) and sampled in phylogenetic analysis. The nLSU dataset, also including three nLSU sequences of $P$. sulphurascens, three of $P$. weirii, and one of $P$. ferrugineofuscum, resulted in an alignment with 912 characters. The ML tree tested by 650 BS replicates (Fig. 2) showed that the pathogenic fungus on $S$. przewalskii formed a distinct lineage that was separated from $P$. sulphurascens and $P$. weirii, and had a closer relationship with $P$. weirii than with $P$. sulphurascens.

Pathogenicity tests. The four fungal isolates of $P$. qilianense that we used to inoculate roots of 1-year-old S. przewalskii seed-

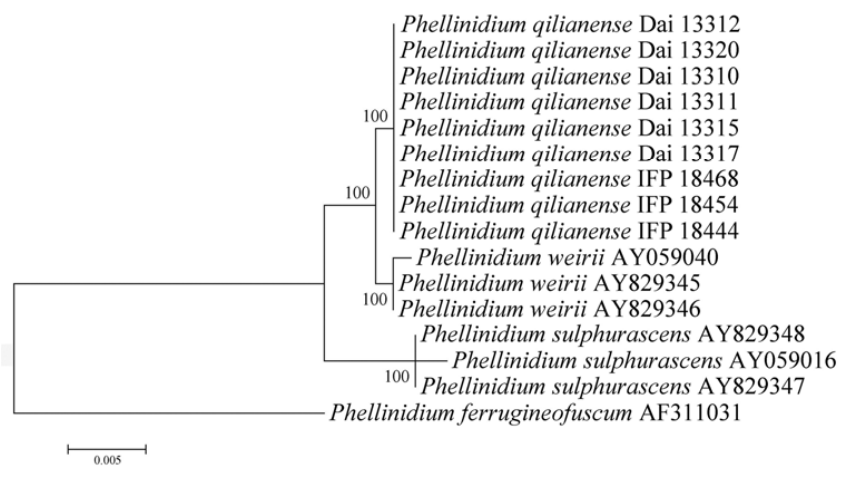

Fig. 2. Phylogenetic relationships among Phellinidium qilianense (in bold), P. sulphurascens and $P$. weirii.

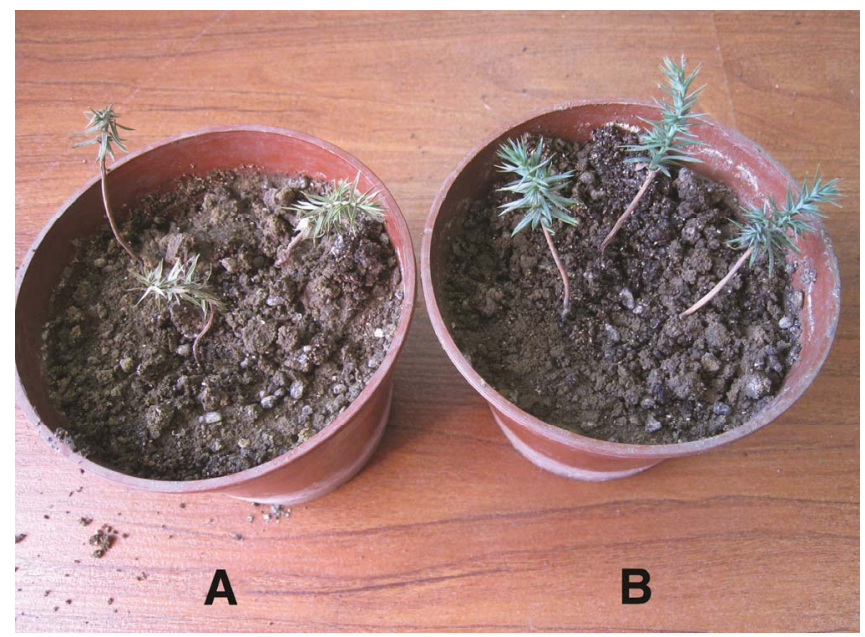

Fig. 3. A, Symptoms of one year old seedlings of Sabina przewalskii infected by Phellinidium qilianense Dai 13309. B, Control. lings colonized roots within 2 months, as evidenced by seedlings showing distinct chlorotic foliage (Fig. 3). Isolates of $P$. qilianense that we obtained from the decayed roots of inoculated seedlings (Fig. 4) were the same as those we used to inoculate seedlings, based on colony morphology (brownish generative hyphae with simple septa and plenty of hyphoid setae with sharp tips) and nLSU sequences. Recovery frequency from the decayed seedling roots was $88.8 \%$ for P. qilianense, $11.2 \%$ for Fusarium sp. and other fungi, and $0 \%$ for control treatment.

Taxonomic description. Phellinidium qilianense B.K. Cui, L.W. Zhou \& Y.C. Dai, sp. nov. Figures 1D \& 5.

MycoBank: MB 808449

Basidiocarps perennial, resupinate, inseparable, soft corky, without odor or taste when fresh, becoming corky to fibrous upon drying, up to $30 \mathrm{~cm}$ long, $14 \mathrm{~cm}$ wide, and $3 \mathrm{~cm}$ thick at center. Pore surface grayish brown to honey-yellow; margin cinnamonbuff, up to $5 \mathrm{~mm}$ wide; pores round or angular, 5 to 8 per $\mathrm{mm}$; dissepiments thick, entire, or slightly lacerate. Subiculum cinnamon-buff, corky, very thin, about $1 \mathrm{~mm}$ thick. Tubes yellowish brown to cinnamon-buff, fibrous, new tubes distinctly paler contracting with old one, old tubes yellowish brown; tube layers up to $29 \mathrm{~mm}$ long, indistinctly stratified.

Hyphal system monomitic; generative hyphae simple septate; tissues darkening but otherwise unchanged in KOH. Subiculum. Generative hyphae hyaline to yellowish, thin- to slightly thickwalled, frequently branched and septate, interwoven, 2 to $5.5 \mu \mathrm{m}$ in diameter. Hymenophoral trama. Generative hyphae hyaline to yellowish, thin- to slightly thick-walled, occasionally branched, frequently septate, parallel along the tubes, 2.5 to $4.5 \mu \mathrm{m}$ in diameter. Hyphoid setae occasionally in subiculum, dominant in trama, dark brown, subulate, sharp-pointed, thick-walled with a narrow lumen, originating from the simple septum of yellowish, slightly thickwalled hyphae, parallel along the tubes, straight, occasionally penetrating into hymenium, sometimes apically to centrally encrusted by small rosettes of crystals, several hundreds of $\mu \mathrm{m}$ long, 5 to 10 $\mu \mathrm{m}$ in diameter. Hymenial setae absent. Cystidia hyaline, subulate,

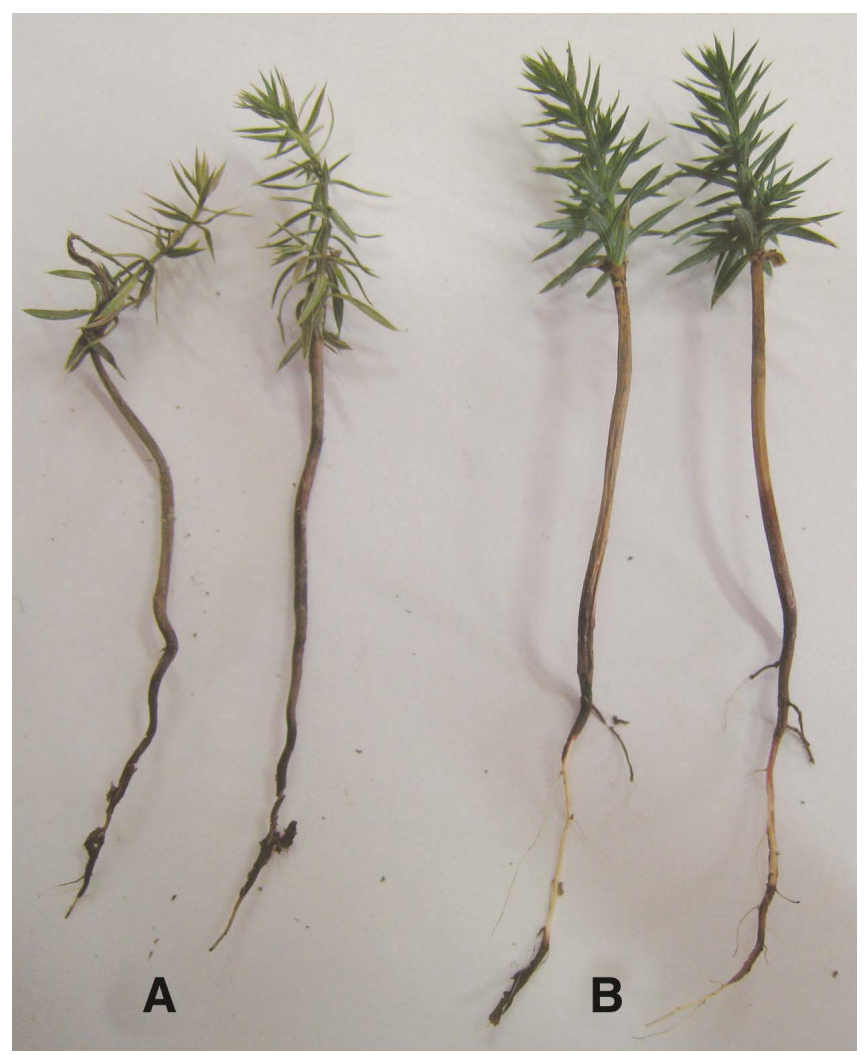

Fig. 4. A, Symptoms on roots of one year old seedlings of Sabina przewalskii infected by Phellinidium qilianense Dai 13309. B, Control. 
thin-walled, sharp-pointed, 17 to $30 \times 3$ to $5 \mu \mathrm{m}$; cystidioles absent. Basidia barrel-shaped, thin-walled, with four sterigmata and a simple septum at the base, 8 to $12 \times 4$ to $6 \mu \mathrm{m}$; basidioles in shape similar to basidia, but slightly smaller. Basidiospores hyaline, broadly ellipsoid, thin-walled, smooth, IKI-, CB-, (3.5-) 3.7 to 4.3 $(-4.4) \times 2.8$ to $3.3(-3.5) \mu \mathrm{m}, \mathrm{L}=3.93, \mathrm{~W}=3.06, \mathrm{Q}=1.27$ to 1.3 $(n=60 / 2)$.

Etymology: Qilianense (Lat.): referring to the type locality, Qilian Mountains.

Materials studied. China. Qinghai: Menyuan County, Xianmi Forest Farm, Sigou, on base of living S. przewalskii, 7 September 2012, Yuan 6424 (holotype in IFP 18444); on base of dying $S$. przewalskii, Yuan 6441 (paratype in IFP 18454); Donghai, on fallen trunk of S. przewalskii, 7 September 2012, Yuan 6458 (paratype in IFP 18468). Xianfeng, on base of living $S$. przewalskii, 28 July 2013, Dai 13304 (paratype in BJFC 14792); on base of dead $S$. przewalskii, 28 July 2013, Dai 13310 (paratype in BJFC 14793); on fallen trunk of S. przewalskii, 28 July 2013, Dai 13320 (paratype in BJFC 14795).

\section{Discussion}

Laminated root rot of $S$. przewalskii was first found in the Xianmi Forest Farm, Qinghai Province, northwest China, about 20 years ago according to the local forestry department. The pathogen responsible for the disease was initially identified without molecular study as $P$. weirii $(2,5)$. The present molecular analysis showed $P$. weirii and $P$. qilianense were in two distinct clades. Some samples of $P$. weirii from North America were also studied and morphological differences between the two species were found. Basidiospores of $P$. weirii are distinctly larger than those in $P$. qilianense $(4.9$ to $5.3 \times 3.2$ to $3.8 \mu \mathrm{m}$ vs 3.7 to $4.3 \times 2.8$ to $3.3 \mu \mathrm{m}$ ). In addition, $P$. weirii occurs mainly on Thuja plicata in North America, while P. qilianense grows exclusively on $S$. przewalskii in northwest China.

$P$. sulphurascens is another pathogen on conifers in northeast, northwest, and southwest China $(3,5)$, and it also causes a laminated root rot. Although $P$. sulphurascens has a wider distribution than $P$. qilianense, it mostly occurs in mature trees within old natural forests. According to our field investigation, $P$. sulphurascens did not cause serious disease on gymnosperm trees in China, and no distinct disease patches or root rot centers were found caused by this fungus. $P$. sulphurascens has an annual habit and larger pores (4 to 5 per $\mathrm{mm}$ ), and attacks species of the Pinaceae, while $P$. qilianense has a perennial habit, smaller pores ( 5 to 8 per $\mathrm{mm}$ ), and

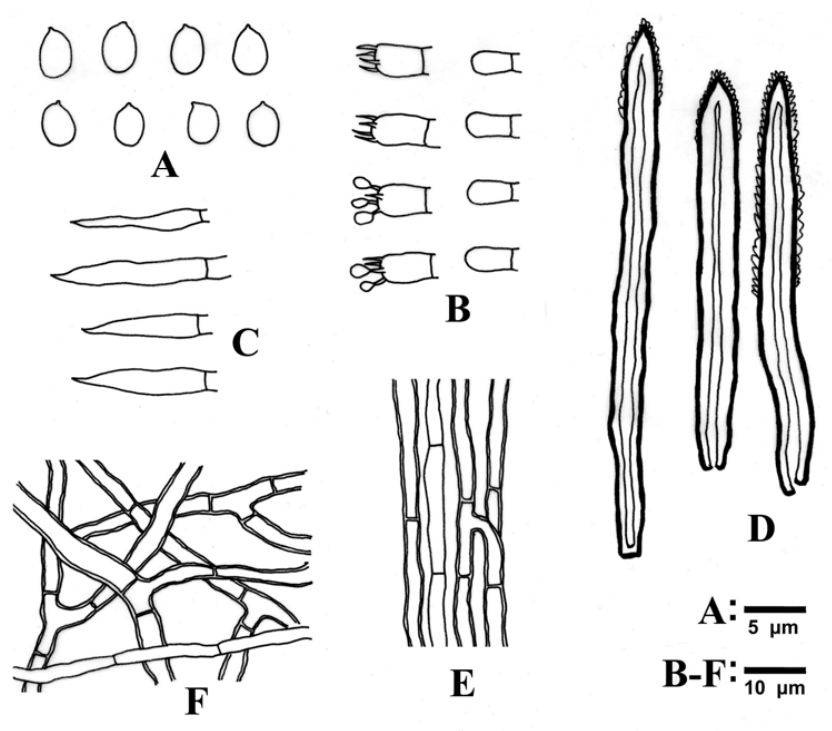

Fig. 5. Microscopic structures of Phellinidium qilianense (drawn from specimen IFP 18444, holotype). A, Basidiospores. B, Basidia and basidioles. C, Cystidia. D, Hyphoid setae. E, Hyphae from trama. F, Hyphae from subiculum. infects species of Cupressaceae. Besides the morphology, the two species have different ecology; $P$. sulphurascens prefer moist environments, while $P$. qilianense favors dryer sites.

Based on phylogenetic analysis inferred from nLSU sequences, all nine pathogenic samples formed a distinct lineage with a high degree of support. They definitely belong to Phellinidium, and a new name, $P$. qilianense, is proposed in this paper. $P$. qilianense was found to be more closely related to $P$. weirii than any other species in the genus.

Laminated rot of roots caused by $P$. qilianense in natural forests of S. przewalskii was found in the Qilian Mountains of Qinghai Province. Many distinct disease patches were observed varying in size but usually about a few hectares. The characteristics of diseased trees are slow growth, thin crowns, chlorotic foliage, and tree decline. Although the number of infected trees in the plots was 40 to $90 \%$, it may actually be found in greater amounts since it is difficult to diagnose during early stages of infection where neither symptoms nor fruiting body of the fungus are evident. During the early stages of the decay caused by $P$. qilianense, the wood color is almost the same as the wood in healthy tree, while in the advanced stages of decay, the wood separated along the annual ring because of localized attack within the annual rings. Usually the wood became softer, paler, and lighter than healthy wood, showing characteristics for laminated rot.

P. qilianense is an aggressive pathogen on $S$. przewalskii in the Qilian Mountains, northwest China. Although some trees may be infected for more than 20 years and show decline, the fungus can also kill trees. Pathogenicity testing was not done on mature trees in the field because it may take many years to get the results. Instead, small seedlings were inoculated in the greenhouse to demonstrate pathogenicity. The laminated root rot on Qilian juniper has been increasing in severity in recent years, and it appears that the disease was spread vegetatively from root to root. Further studies on disease progression, pathogenicity tests on trees in the field, and disease management shall be done in the future.

\section{Acknowledgments}

We express our gratitude to Mr. Jian-Hai Ma (the forest Pest Control Department of Qinghai Province, China) for helping in field investigation and to Prof. Robert A. Blanchette (University of Minnesota, United States) for improving the manuscript. The research was financed by the Fundamental Research Funds for the Central Universities (Project No. JC2013-1) and the National Natural Science Foundation of China (No. 31200015).

\section{Literature Cited}

1. Banik, M. T., Paul, J. A., Burdsall, H. H., and Cooke, M. E. 1993. Serological differentiation of two forms of Phellinus weirii. Mycologia 85:605-611.

2. Dai, Y. C. 2004. First report of laminated root rot on Sabina przewalskii caused by Phellinus weirii sensu stricto in China. Plant Dis. 88:573.

3. Dai, Y. C. 2010. Hymenochaetaceae (Basidiomycota) in China. Fungal Divers. 45:131-343.

4. Dai, Y. C. 2012. Polypore diversity in China with an annotated checklist of Chinese polypores. Mycoscience 53:49-80.

5. Dai, Y. C., Cui, B. K., Yuan, H. S., and Li, B. D. 2007. Pathogenic wooddecaying fungi in China. For. Pathol. 37:105-120.

6. Dai, Y. C., and Qin, G. F. 1998. Phellinidium sulphurascens-a forest pathogen in China. Fungal Sci. 13:101-107.

7. Gilbertson, R. L., and Ryvarden, L. 1987. North American polypores 2. Fungiflora, Oslo.

8. Guindon, S., and Gascuel, O. 2003. A simple, fast and accurate method to estimate large phylogenies by maximum-likelihood. Syst. Biol. 52:696-704.

9. Hansen, E. M., Angwin, P. A., Dreisbach, T. A., Gernandt, D., and McWilliams, M. G. 1998. Species limits for Phellinus weirii. Pages 119127 in: Root and Butt Rots of Forest Tress, 9th International Conference on Root and Butt Rots, Carcans-Maubusisson, France.

10. Hattori, T., Abe, Y., and Usugi, T. 1996. Distribution of clones of Phellinus noxius in a windbreak on Ishigaki Island. Eur. J. For. Pathol. 26:69-80.

11. Katoh, K., Misawa, K., Kuma, K., and Miyata, T. 2002. MAFFT: a novel method for rapid multiple sequence alignment based on fast Fourier transform. Nucleic Acids Res. 30:3059-3066.

12. Katoh, K., and Toh, H. 2008. Recent developments in the MAFFT multiple sequence alignment program. Brief Bioinform. 9:286-298.

13. Larsen, M. J., Lombard, F. F., and Clark, J. W. 1994. Phellinus sulphurascens and the closely related $P$. weirii in North America. Mycologia 86:121-130.

14. Lim, Y. W., Yeung, Y. C. A., Sturrock, R., Leal, I., and Breuil, C. 2005. 
Differentiating the two closely related species, Phellinus weirii and $P$. sulphurascens. For. Pathol. 35:305-314.

15. Murrill, W. A. 1914. An enemy of the western red cedar. Mycologia 6:9394

16. Pattengale, N. D., Alipour, M., Bininda-Emonds, O. R. P., Moret, B. M. E., and Stamatakis, A. 2010. How many bootstrap replicates are necessary? J. Comput. Biol. 17:337-354.

17. Posada, D. 2008. jModelTest: Phylogenetic model averaging. Mol. Bio. Evol. 25:1253-1256.

18. Silvestro, D., and Michalak, I. 2012. raxmlGUI: a graphical front end for RAxML. Org. Divers. Evol. 12:335-337.
19. Sinclair, W. A., Lyon, H. H., and Johnson, W. T. 1987. Diseases of Trees and Shrubs. Cornell University Press, Ithaca, NY.

20. Stamatakis, A. 2006. RAxML-VI-HPC: maximum likelihood-based phylogenetic analyses with thousands of taxa and mixed models. Bioinformatics 22:2688-2690.

21. Vilgalys, R., and Hester, M. 1990. Rapid genetic identification and mapping of enzymatically amplified ribosomal DNA from several Cryptococcus species. J. Bacteriol. 172:4238-4246.

22. Wagner, T., and Fischer, M. 2002. Proceedings towards a natural classification of the worldwide taxa Phellinus s.l. and Inonotus s.l., and phylogenetic relationships of allied genera. Mycologia 94:998-1016. 